\title{
Conductance fluctuations in systems with a random-magnetic-field scattering
}

\author{
Vladimir I. Fal'ko \\ Max-Planck-Institut für Festkörperforschung, Heisenbergstrasse 1, 70569 Stuttgart, Germany \\ and Institute of Solid State Physics, Russian Academy of Science, Chernogolovka, 142432, Russia
}

(Received 5 August 1994)

\begin{abstract}
The perturbation-theory study of universal conductance fluctuations is generalized to systems with electron scattering in a static random magnetic field. Our calculation confirms the intuitive expectation that the amplitude of fluctuations coincides with the universal value typical for the unitary random-scattering ensemble. An application of this result to micrometer-size wires with the twodimensional electron liquid near filling factor $\nu=1 / 2 q$ allows us to predict aperiodic Coulomb-type oscillations in this system beyond the Coulomb-blockade regime, which would manifest a correspondence between the Aharonov-Bohm and Aharonov-Casher effects specific to composite fermions.
\end{abstract}

\section{INTRODUCTION}

The features of electron transport in a nonuniform or random magnetic field (RMF) have been intensively studied during the past few years. ${ }^{1-6}$ Theoretical activities in this direction have been stimulated partly by experimental efforts to produce inhomogeneous magnetic fields in high-mobility two-dimensional (2D) semiconductors using external means: surface superconducting ${ }^{7-9}$ or magnetic ${ }^{10}$ layers, or an application of a magnetic field parallel to a slightly curved heteroboundary. ${ }^{11,12}$ But mainly, attention has been drawn to systems with RMF scattering by recent ${ }^{13,14}$ single-particle formalism to describe strongly correlated $2 \mathrm{D}$ electrons at filling factors around $\nu=1 / 2 q$ in terms of free quasiparticles moving in a renormalized built-in magnetic field. According to the composite-fermion theory of the fractional quantum Hall effect, ${ }^{13,14}$ in the region of real magnetic fields where $\nu \approx \frac{1}{2}$, the liquid of electrons is equivalent to an ideal Fermi gas of quasiparticles (each associated with the charge $e$ and two attached flux quanta, 2 $\Phi_{0}$ ) moving in an effective magnetic field $H_{\text {eff }}=H-H_{1 / 2}\left(n_{e}\right)$. The latter is determined both by the external field itself and by the local electron density $\left(H_{1 / 2}=4 \pi n_{e} \hbar c / e\right)$, so that the real electron scattering on a smooth screened random potential and in a high external magnetic field can be converted into the free quasiparticle scattering on a weak built-in effective RMF. ${ }^{14,15}$

Recent theories of electrons in a RMF have concentrated on the studies of the localization problem. Although different authors propose different scenarios of the localization itself, conclusions drawn from the results of all the methods ${ }^{1-4,6}$ agree at the following point: Even if the particle states are localized at $T=0$, the localization length $L_{c}$ can be long enough $\left(L_{c} \gg l \gg \hbar / p_{F}\right)$ to expect (at a finite temperature) a wide region of metallic conductivity where quantum localization corrections are reduced and the transport shows a dominantly classical behavior.

Nevertheless, it is known that, despite magnetic field suppression of the weak localization in macroscopic systems, ${ }^{16}$ quantum interference effects are still observable in small structures. The sample-specific interference pattern of electron waves produces universal conductance fluctuations ${ }^{17,18}$ at the scale of a quantum $e^{2} / h$. One can assume that the same takes place in a confined system with random-magnetic-field scattering. In what follows, we study conductance fluctuations in conductors where all the disorder is due to an irregular magnetic field. We consider the metallic regime since this one has been discussed in the application to the existing experimental conditions; in particular, the authors of Refs. 15 and 19 report the mean-free path $l$ of composite fermions consistent with the value of the metallicity parameter $p_{F} l / \hbar \sim 10-50$. These calculations are described in Sec. II. The magneto-conductance fluctuations obtained look the same as universal fluctuations ${ }^{17,18,20}$ in a disordered metal subjected to a strong magnetic field and confirm an intuitive expectation that the system with a RMF scattering is a typical representative of the generalized unitary random scattering ensemble.

On the other hand, the relation between the flux $2 \Phi_{0}$ and the electron charge assigned to a quasiparticle in the composite-fermion liquid near the filling factor $\nu=\frac{1}{2}$ has to be taken into account. The arguments as to how that can be done are presented in Sec. III. The abovementioned correspondence forces us to expect fluctuations not only due to a change of real magnetic flux through a sample (as has been recently observed by Simmons et al. ${ }^{21}$ in a microstructure with a $2 \mathrm{D}$ gas at $\nu \approx \frac{1}{2}$ ), but also under such gate voltage variation that implants each additional charge $\delta Q \sim \frac{1}{2} e$ into the sample area. Roughly speaking, this could look like aperiodic Coulomb oscillations if one would forget that they take place beyond the Coulomb-blockade regime. The conductance behavior near other higher even-denominator fractions, $\nu=1 / 2 q$, should be similar, but with an important correction: The multiple flux $2 q \Phi_{0}$ assigned to composite fermions at $\nu=1 / 2 q$ shortens the scale of a charge (i.e., gate voltage) dependence down to the value of $\delta Q \sim \frac{e}{2 q}$. We propose to use this feature as a test of the nontrivial internal structure of elementary quasiparticles predicted 
by the composite-fermion model of the fractional quantum Hall effect.

\section{PERTURBATION-THEORY CALCULATIONS}

From the technical side, our analysis is based on the perturbation theory, a convenient tool to study the transport in the metallic regime. As compared to the diagrammatic calculations described in Ref. 16, the technique has to be adopted in order to include the electron scattering on a random vector potential (instead of usual impurity scattering) and to account for a strong anisotropy of scattering amplitudes, in particular, the dominance of a low-angle scattering. The main part of what we do below consists of the derivation of two-particle Green's functions.

As the first step, we replace scalar disordered potential vortices in the perturbation-theory calculations ${ }^{16}$ by an interaction $\delta u=\mathbf{v} \frac{e a}{c}$ with a planar component of a random vector potential $\mathbf{a} ; \mathbf{v}$ is the velocity operator. In the plane-wave representation, $\mathbf{v}=\left(\mathbf{p}+\mathbf{p}^{\prime}\right) / 2 m$. The fluctuations of the random field a can be described using the diagonal gauge, $\left\langle a_{\alpha} a_{\beta}\right\rangle_{\mathbf{q}}=\delta_{\alpha \beta} f(q) ;^{2}$ it is easy to show that the transverse part, $q_{\alpha} q_{\beta} / q^{2}$, of the correlation function $\left\langle a_{\alpha} a_{\beta}\right\rangle_{\mathbf{q}}$ does not affect the transport properties of electrons. In the case when a random field in the $2 \mathrm{D}$ system is produced by a thin film of a magnetic material sputtered on the semiconductor surface at the distance $d$ fron the 2D gas, the fluctuation of a gauge field is described by the correlation function of the above fornı with $f(q)=e^{-2 q d}\left\langle\mu^{2}\right\rangle$. When a magnetic powder fills one half of a space, $f(q)=q^{-1} e^{-2 q d}\left\langle\mu^{2}\right\rangle$. The correlation function $f(q)$ due to a magnetic field frozen into a superconducting gate as a number of residual fluxes corresponds to $f(q)=q^{-2} e^{-2 q d}\left\langle\mu^{2}\right\rangle$. In the above definitions, $\left\langle\mu^{2}\right\rangle$ is the quantity proportional to the rms value of distributed magnetic moments and could be referred to as a phenomenological parameter. Finally, a built-in magnetic disorder associated with a source of scattering in the composite-fermion system can be described by the latter expression with $d=0$.

After averaging over a magnetic disorder, the vertex

$$
\begin{aligned}
\Gamma^{(0)}\left(\mathbf{p}, \mathbf{p}^{\prime} ; \mathbf{p}^{\prime \prime}, \mathbf{p}^{\prime \prime}\right. & \left.+\mathbf{p}-\mathbf{p}^{\prime}\right) \\
& =w \frac{\left(\mathbf{p}+\mathbf{p}^{\prime}\right) \cdot\left(2 \mathbf{p}^{\prime \prime}+\mathbf{p}-\mathbf{p}^{\prime}\right)}{4 p_{F}^{2}} f\left(\left|\mathbf{p}-\mathbf{p}^{\prime}\right|\right)
\end{aligned}
$$

appears as a construction unit of the perturbation-theory diagrams $\left[w=\left(e v_{F}\right)^{2}\right]$. In the figures below, this is shown by dashed lines. Due to the isotropy of the system, which is held in average, one should distinguish two nonrenormalized "dashed" vortices,

$$
\begin{aligned}
& \Gamma_{0}^{(0)}=\int \frac{d \Omega_{\mathbf{p}^{\prime}}}{2 \pi} \Gamma^{(0)}\left(\mathbf{p}, \mathbf{p}^{\prime} ; \mathbf{p}, \mathbf{p}^{\prime}\right) \\
& \Gamma_{1}^{(0)}=\int \frac{d \Omega_{\mathbf{p}^{\prime}}}{2 \pi} \frac{\mathbf{p} \cdot \mathbf{p}^{\prime}}{p_{F}^{2}} \Gamma^{(0)}\left(\mathbf{p}, \mathbf{p}^{\prime} ; \mathbf{p}, \mathbf{p}^{\prime}\right) .
\end{aligned}
$$

The first $\Gamma_{0}^{(0)}$ plays the role in determining the collision rate $\tau^{-1}$ which enters into the retarded (advanced) singleparticle Green's function,

$$
G^{R, A}(\mathbf{p})=\frac{1}{\epsilon-\epsilon(p) \pm i \tau^{-1} / 2}, \quad \tau^{-1}=2 \pi \nu_{F} \Gamma_{0}^{(0)}
$$

Depending on the origin of a random magnetic field, this rate can strongly differ from the momentum relaxation rate and, in some cases [when $f(q) \propto q^{-2}$ ], it formally diverges. Nevertheless, this does not mean that the perturbation theory is not applicable to studies of the transport in this system. Even in the case of a divergent $\tau^{-1}$, the momentum relaxation rate, $\tau_{p}^{-1}=2 \pi \nu_{F}\left(\Gamma_{0}^{(0)}-\Gamma_{1}^{(0)}\right)$, is finite ${ }^{1}$ and the electron mean-free path $l=v_{F} \tau_{p}$ can be long enough to provide a metallicity parameter $p_{F} l \gg 1$. In what follows, we operate with vertices $\Gamma_{0,1}^{(0)}$ as if they are finite and find that the quantity $\tau$ cancels from the transport coefficients, whereas only the convergent $\tau_{p}^{-1}$ remains, which approves this approach.

As we know from the theories of an ordinary potential scattering, ${ }^{16}$ there are two main constraints that play the dominant role in diagrammatic calculations of such quantities as the conductivity, the localization corrections to it, and the correlation function of mesoscopic conductances. These are the renormalized disordered vertex $\Gamma$ and the renormalized current vertex $\mathbf{V}$, both being the sums of the diagrams shown in Fig. 1.

In calculating the renormalized vortex $\Gamma\left(\mathbf{p}, \mathbf{p}^{\prime} ; \mathbf{p}^{\prime \prime}, \mathbf{p}^{\prime \prime \prime}\right)$ we should distinguish particle-hole and particle-particle channels. The two-particle Green's function in the particle-hole channel (diffusion) is related to the diffusion of density and is expected ${ }^{16}$ to possess a pole in the region of small transferred momenta. That is why we calculate the function $\Gamma_{d}\left(\mathbf{p}, \mathbf{p}^{\prime} ; \mathbf{Q}\right)=\Gamma\left(\mathbf{p}+\mathbf{Q} / 2, \mathbf{p}^{\prime}+\right.$ $\left.\mathbf{Q} / 2 ; \mathbf{p}-\mathbf{Q} / 2, \mathbf{p}^{\prime}-\mathbf{Q} / 2\right)$ assuming that $Q \ll p, p^{\prime} \sim p_{F}$. The two-electron Green's function in the particle-particle channel (Cooperon) is related to the diffusion of a coherent particle phase ${ }^{16}$ and (in systems with the timereversal symmetry) is formed by the phase space region of small summed momenta: $\Gamma_{c}\left(\mathbf{p}, \mathbf{p}^{\prime} ; \mathbf{Q}\right)=\Gamma\left(\mathbf{p}+\mathbf{Q} / 2, \mathbf{p}^{\prime}+\right.$ $\left.\mathbf{Q} / 2 ;-\mathbf{p}+\mathbf{Q} / 2,-\mathbf{p}^{\prime}+\mathbf{Q} / 2\right)$. Since the electron scattering by a RMF is strongly anisotropic and in the most interesting cases is a dominantly low angle, we use the trick often applied to kinetic equations in systems with an anisotropic scattering. We represent $\Gamma_{d(c)}$ in the form of a multipolar expansion series,

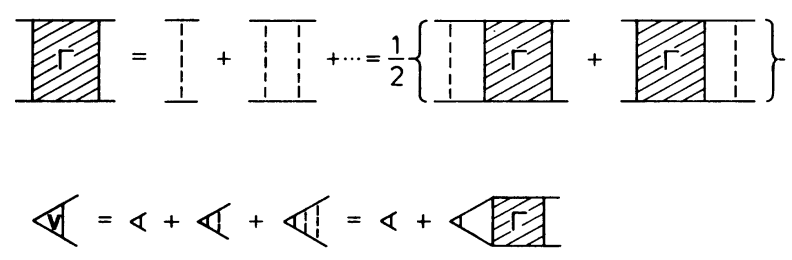

FIG. 1. Diagrammatic representation of vortices $\mathbf{V}$ and $\Gamma$ and the corresponding Dyson's equations on them. The free-electron current vertex (open triangle) equals $v_{F}$. Dashed lines denote the initial magnetic disorder vortex $\Gamma^{(0)}$. 


$$
\begin{aligned}
\Gamma_{d(c)}(\mathbf{Q})= & \Gamma_{d(c) 0}(\mathbf{Q})+\left(\frac{\mathbf{p} \cdot \mathbf{p}^{\prime}}{p_{F}^{2}}\right) \Gamma_{d(c) 1}(\mathbf{Q}) \\
& +\left(\frac{\left(\mathbf{p}+\mathbf{p}^{\prime}\right) \cdot \mathbf{Q}}{2 p_{F}^{2}}\right) \Pi_{d(c)+}(\mathbf{Q}) \\
& +\left(\frac{\left(\mathbf{p}-\mathbf{p}^{\prime}\right) \cdot \mathbf{Q}}{2 p_{F}^{2}}\right) \Pi_{d(c)-}(\mathbf{Q})+\cdots
\end{aligned}
$$

and integrate both sides of the Dyson equation on $\Gamma$ over the orientation of $\mathbf{p}$ and $\mathbf{p}^{\prime}$ with the multipolar weights. After keeping leading terms, we find that in the particlehole channel

$$
\Gamma_{d 0}=\Gamma_{0}^{(0)}+\left(1-i \omega \tau-\frac{\left(v_{F} \tau Q\right)^{2}}{2}\right) \Gamma_{d 0}+i \frac{v_{F} \tau Q^{2}}{4 p_{F}} \Pi_{d+},
$$

$\Pi_{d+}=i \frac{v_{F} \tau p_{F}}{2}\left(2 \Gamma_{d 0} \frac{\Gamma_{1}^{(0)}}{\Gamma_{0}^{(0)}}+\Gamma_{d 1}\right)+\frac{1}{2}\left(1+\frac{\Gamma_{1}^{(0)}}{\Gamma_{0}^{(0)}}\right) \Pi_{d+}$,

and

$$
\frac{1}{2} \Gamma_{d 1} \approx \Gamma_{1}^{(0)}+\pi \nu_{F} \tau \Gamma_{1}^{(0)} \Gamma_{d 1}, \quad \Pi_{d-} \approx 0 .
$$

From these equations, one can easily conclude that the diffusion modes $\Gamma_{d 0}$ and $\Pi_{d+}$ are gapless and contain a pole in the space of variables $\mathbf{Q}$ and $\omega$, whereas the mode $\Gamma_{1}$ does not show any long wavelength contribution, and $\Gamma_{1}=2 \Gamma_{0}^{(0)} \Gamma_{1}^{(0)} /\left(\Gamma_{0}^{(0)}-\Gamma_{1}^{(0)}\right)$. This immediately eliminates $\Gamma_{1}$ from the first two equations in Eq. (2) and leads us to the diffusion equation on the vertex $\Gamma_{d 0}$,

$$
\Gamma_{d 0}=\frac{P(\mathbf{Q})}{2 \pi \nu_{F} \tau^{2}}, \quad\left(-i \omega-D Q^{2}\right) P=1,
$$

where $D=v_{F}^{2} /\left[4 \pi \nu_{F}\left(\Gamma_{0}^{(0)}-\Gamma_{1}^{(0)}\right)\right]=v_{F}^{2} \tau_{p} / 2$ is a diffusion coefficient and

$$
\begin{aligned}
\tau_{p}^{-1} & =2 \pi \nu_{F}\left(\Gamma_{0}^{(0)}-\Gamma_{1}^{(0)}\right) \\
& =2 \pi \nu_{F} \int \frac{d \Omega_{\mathbf{p}^{\prime}}}{2 \pi}\left(1-\frac{\mathbf{p} \cdot \mathbf{p}^{\prime}}{p_{F}^{2}}\right) \Gamma^{(0)}\left(\mathbf{p}, \mathbf{p}^{\prime}\right)
\end{aligned}
$$

is the momentum relaxation rate.

Naturally, for systems with a broken time-reversal symmetry, the vortex $\Gamma_{c}$ in the particle-particle channel does not show any singular contribution:

$$
\Gamma_{c 0}=-\frac{1}{2} \Gamma_{0}^{(0)}, \quad \Gamma_{c 1}=-2 \Gamma_{1}^{(0)} \Gamma_{0}^{(0)} /\left(\Gamma_{0}^{(0)}+\Gamma_{1}^{(0)}\right) .
$$

This manifests in the fact that the coherence between two time-reversed trajectories is destroyed at the time scale of a scattering time $\tau$ and that, in average, the electron behavior is more close to that of a classical particle, as has been discussed in Ref. 1 .

The renormalization of a current vortex $\mathbf{V}(\mathbf{p})=V \underset{p_{F}}{\mathbf{p}}$, can be derived from its relation to the already calculated term $\Gamma_{d 1}, V=v_{F}\left(1+\pi \nu_{F} \tau \Gamma_{d 1}\right)$. After substituting the result of Eqs. (2) we arrive at

$$
V=v_{F} \frac{\Gamma_{0}^{(0)}}{\Gamma_{0}^{(0)}-\Gamma_{1}^{(0)}}=\frac{v_{F} \tau_{p}}{\tau} .
$$

At this stage, we can already calculate the transport coefficients of the system. In particular, the Einstein relation between the diffusion coefficient $D$ due to the electron scattering on a RMF and the conductivity $\sigma$. $\sigma=e^{2} \nu_{F} D=\frac{s e^{2}}{2 h}\left(p_{F} l / \hbar\right)$, can be easily reproduced, as well as the value of the Hall coefficient ( $s$ is the spin degeneracy of the Fermi surface). From the suppression of the Cooperon channel, one can see that the first logarithmic quantum corrections to the conductivity ${ }^{16}$ $\left[\frac{e^{2}}{2 \pi h} \ln \left(L_{\phi} / l\right)\right]$ is absent. After some algebra, one can find that the logarithmic localization correction can appear only in the next order with respect to the inverse metallicity parameter $1 / p_{F} l$ as $\delta \sigma_{l o c}=-\kappa \frac{e^{2}}{2 \pi h} \frac{\ln (L / l)}{p_{F} l}$. This statement repeats earlier predictions ${ }^{3}$ and manifests that the system has the localization properties of a unitary random scattering ensemble studied in general localization theories. ${ }^{22}$ The exact value of a coefficient $\kappa$ can be derived much more elegantly ${ }^{6}$ after reducing the problem to the nonlinear supersymmetric $\sigma$ model. $^{22}$

As we mentioned above, the suppression of localization corrections to conductivity does not mean that the electron transport in systems with RMF scattering necessarily loses all its quantum features. Just as in disordered conductors in a strong magnetic field, ${ }^{18}$ the samplespecific interference pattern formed by diffusive electron waves is still present if the temperature is low enough to prevent phase-breaking processes. Therefore, the $2 \mathrm{D}$ electron gas subjected to a random magnetic field has to show the conductance fluctuations, similarly to the electrons in disordered conductors. Within the frame of a perturbation theory, mesoscopic conductance fluctuations are described by the mean square of the deviation of a sample-specific conductance from the averaged value, $\left\langle\delta G^{2}\right\rangle$. The set of diagrams that represent this quantity (see Fig. 2) has been derived in earlier works. ${ }^{23}$ Skipping the details, the magnitude of the effect is controlled by the gapless diffusive mode, and thus, the ladder part
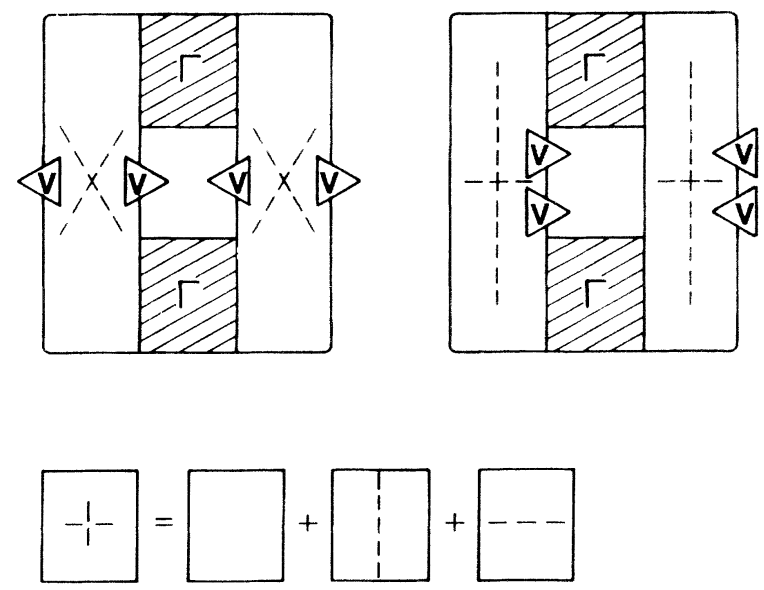

FIG. 2. Standard perturbation theory diagrams that describe the mean square of conductance fluctuations in a unitary ensemble. Inset explains the meaning of "dashed crosses." Under conditions discussed in the text, the second diagram gives one-half of the first. 
of these graphs is dominated by the renormalized vertex $\Gamma_{d 0}(\mathbf{Q})$ given by Eq. (2). The collision time $\tau$ (which primarily entered into the expressions for $\Gamma_{d 0}$ and the current vortex $\mathbf{V}$ ) cancels, and the rms value of conductance fluctuations in a RMF expressed in terms of a diffusive mode $P(\mathbf{Q})$,

$$
\left\langle\delta G^{2}\right\rangle=6\left(\frac{e^{2}}{h} \frac{D}{L^{2}}\right)^{2} \sum_{Q}[P(\mathbf{Q})]^{2}
$$

takes exactly the same form as in a usual disordered conductor subjected to a strong magnetic field, independently of the specific form and origin of the disordered static vector potential a.

The calculation of $\left\langle\delta G^{2}\right\rangle$ needs the diffusion equation in (3) to be completed with the boundary conditions. To be consistent, the latter should be zero at hard walls $\left(\mathbf{r}=\mathbf{r}_{s}\right), \nabla P\left(\mathbf{r}_{s}\right)=0$, and the loss of a coherent phase memory in bulk electrodes $\left(\mathbf{r}=\mathbf{r}_{L}\right)$ gives $P\left(\mathbf{r}_{L}\right)=0 .{ }^{24}$ To be specific, we distinguish a long (quasi-1D) wire and find

$$
\left\langle\delta G^{2}\right\rangle=\frac{s}{15}\left(\frac{e^{2}}{h}\right)^{2},
$$

which also would describe the amplitudes of aperiodic conductance oscillations under variations of an additional external homogeneous magnetic field.

In microstructures prepared from extremely pure 2D electron gas subjected to a combination of a random (a) and a homogeneous $(H)$ magnetic field, Eq. (6) confirms a preliminary expectation that the transport in this system should show all the features of a typical representative of a unitary random scattering ensemble (the spindegeneracy factor $s$ can be equal to 2 or 4 , depending on the Zeeman splitting efficiency). On the other hand, the correlation properties of magnetoconductance fluctuations in each of possible candidates for such a system would be different. In most of them, any variation of an external magnetic field brings, at the first instance, a variation of a random magnetic field, too. As applied to the RMF created by a layer of powdered ferromagnetic particles on the surface of a semiconductor, this would come through their polarization. When a RMF is created by a number of flux tubes penetrated through a superconducting screen put over a mesoscopic bridge, ${ }^{9}$ the effect of a homogeneous external field depends on the features of the superconducting material. Therefore, if the RMF is created using external means, the correlations in magnetoconductance fluctuations are strongly affected by properties of these "external means," which makes it difficult to estimate the correlation field in advance. Only when the source of a magnetic-field randomness lies in a roughness or a curvature of the $2 \mathrm{D}$ electron channel imposed on the parallel or strongly inclined magnetic field, ${ }^{11,12}$ can the correlation field be estimated from the direct change of a mean square of a flux penetrated into the sample area: $S\left\langle H_{c}^{2}\right\rangle^{1 / 2} \sim \Phi_{0}$.

\section{CONDUCTANCE FLUCTUATIONS IN COMPOSITE-FERMION LIQUIDS AT $\nu=\frac{1}{2 q}$}

To apply the results of the above calculations to the quantum transport properties of micrometer-size wires with the composite-fermion liquid (the 2D electron gas near the even-denominator filling factors) one should take into account the two following remarks. First, according to Refs. 14 and 15, the transport due to composite quasiparticles is described by their (dissipative) conductivity $\sigma$ which is directly related to observable resistivity $\rho_{x x}$ as $\sigma=1 / \rho_{x x}$, but not by usual inverse-tensor relations. The presence of a Hall term $\rho_{x y}$ is skipped, since the latter is considered as a quantity related to the mean gauge field. Hence, once we discuss the fluctuation of a conductance $G$ due to composite quasiparticles, it has to be imagined as a fluctuation of the inverse resistance $R_{x x}^{-1}$ measured in the four-terminal geometry. Therefore, an estimation in Eq. (6) gives only an order of magnitude of $\delta G=\delta\left(R_{x x}^{-1}\right)$ and should be corrected by geometrical factors specific to the multilead devices. ${ }^{25}$

Second, an application of Eq. (6) to the quantum transport in the composite-fermion system interferes with the unusual correspondence between the Aharonov-Bohm and Aharonov-Casher effects in it and brings us to a conclusion that can be used to demonstrate the multiple flux built up into the composite quasiparticle. Indeed, based on the assumption ${ }^{13,14}$ that the electron system near the filling factor $\nu=\frac{1}{2}$ is equivalent to the Fermi gas of composite quasiparticles that live in an effective magnetic field $H_{\text {eff }}=H-H_{1 / 2}$, we can conclude that the magnetoconductance fluctuations in a microbridge can be equally affected both by the variation of a magneticfield flux through its area or by the change of the electron sheet density.

Conductance fluctuations around $\nu=1 / 2$ under a direct variation of a magnetic field itself have already been observed by Simmons et al.,${ }^{21}$ though the region of interest for the present paper has not been analyzed in detail. On the other hand, the effective magnetic flux through the sample can be varied indirectly, if it is true that each composite quasiparticle ${ }^{13-15,19}$ incorporates two flux quanta attached to a single charge $e$. Therefore, the change of a total effective flux $\Phi_{\text {eff }}=S H_{\text {eff }}$ encircled by a pair of characteristic diffusive trajectories that corresponds to an additional quantum $\Phi_{0}$ can be converted into a density variation, that provides an implantation of an additional charge $\delta Q \sim \frac{e}{2}$ into the contact area $S$. The resulting variations of the conductance versus the gate voltage should look like aperiodic Coulomb oscillations, but this is expected in the system that is open and is definitely beyond the Coulomb-blockade regime.

The same should work for the composite-fermion states near higher even-denominator fillings, $\nu=1 / 2 q$, where each quasiparticle is associated with $2 q$ flux quanta. The latter relation reduces the value of an implanted charge which is sufficient to renew the interference pattern in the sample: down to $\delta Q \sim \frac{e}{2 q}$. Therefore, a comparison of correlation gate voltages extracted from conductance fluctuations near different $\nu=1 / 2 q$ would help one to examine the multiflux internal structure of compos- 
ite fermions. The above proposed experiment would be complementary to the measurements of an effective flux quantum in the fractional quantum Hall effects discussed before. ${ }^{26}$ Its realization requires the temperature $T$ to be below the Thouless energy $E_{c} \sim \pi h D / 2 L^{2}$ for the composite quasiparticles. The latter would be lower than the Thouless energy for real electrons in the same structure at low fields, but the reported values ${ }^{15,19}$ of compositefermion mean-free paths are long enough to expect pronounced features in $\mu \mathrm{m}$-size samples at $T \sim 0.1 \mathrm{~K}$.

\section{CONCLUSIONS}

The above-presented perturbation-theory analysis of conductance fluctuations in systems with electron scat- tering on a static random magnetic field shows that this system possesses all the typical features of a unitary random scattering ensemble. On the other hand, an application of the obtained result to the electron liquid around $\nu=1 / 2 q$ and the unusual correspondence between the Aharonov-Bohm and Aharonov-Casher effects specific to the composite fermions shows a possibility of testing directly the multiple flux structure of composite quasiparticles.

\section{ACKNOWLEDGMENTS}

The author thanks J. Chalker, K. Efetov, S. Feng, K. v. Klitzing, L. Levy, and D. Weiss for discussions and H. Störmer for useful information. Partial support from ISF Grant No. REE-000 is also acknowledged.
${ }^{1}$ B.L. Altshuler and L.B. Ioffe, Phys. Rev. Lett. 69, 2979 (1992).

2 J.M. Wheatley and T.M. Hong, Phys. Rev. B 43, 6288 (1991); D. Khveschenko and S.V. Meshkov, ibid. 47, 12051 (1993).

3 T. Sugiyama and N. Nagaosa, Phys. Rev. Lett. 70, 1980 (1993); Y. Avishai et al., Phys. Rev. B 47, 9561 (1993);

P.A. Lee and D.S. Fisher, Phys. Rev. Lett. 47, 882 (1981).

${ }^{4}$ V. Kalmeyer et al., Phys. Rev. B 48, 11095 (1993); V. Kalmeyer and S.C. Zhang, ibid. 46, 9889 (1992).

${ }^{5}$ D.K.K. Lee and J.T. Chalker, Phys. Rev. Lett. 72, 1510 (1994).

${ }^{6}$ A. Aronov et al., Phys. Rev. B 49, 16609 (1994).

${ }^{7}$ S.J. Bending, K.v. Klitzing, and K. Ploog, Phys. Rev. Lett. 65, 1060 (1990); S.J. Bending et al., Phys. Rev. B 42, 9859 (1990); G.H. Kruithof et al., Phys. Rev. Lett. 67, 2725 (1991).

${ }^{8}$ A.K. Geim et al., Pis'ma Zh. Eksp. Teor. Fiz. 51, 107 (1990) [JETP Lett. 51, 121 (1990)]; Phys. Rev. Lett. 69, 2252 (1992).

${ }^{9}$ A.K. Geim et al., Solid State Commun. 82, 831 (1992).

${ }^{10}$ R. Yagi and Y. Iye, J. Phys. Soc. Jpn. 62, 1279 (1993); D. Weiss (private communication).

${ }^{11}$ V.I. Fal'ko, J. Phys. Condens. Matter 2, 3797 (1990).

12 I.V. Kukushkin (private communication).

13 J.K. Jain, Phys. Rev. Lett. 63, 199 (1989).

${ }^{14}$ B.I. Halperin, P.A. Lee, and N. Read, Phys. Rev. B 47, 7312 (1993).

${ }^{15}$ R.R. Du et al., Phys. Rev. Lett. 70, 2944 (1993); D.R. Leadley et al., ibid. 72, 1906 (1994).

${ }^{16}$ P.A. Lee and T.V. Ramakrishnan, Rev. Mod. Phys. 57, 287 (1985).
${ }^{17}$ C.P. Umbach et al., Phys. Rev. B 30, 4048 (1984); J.C. Licini et al., Phys. Rev. Lett. 55, 2987 (1985).

${ }^{18}$ B.L. Altshuler, Pis'ma Zh. Eksp. Teor. Fiz. 41, 530 (1985) [JETP Lett. 41, 648 (1985)]; P.A. Lee and A.D. Stone, Phys. Rev. Lett. 55, 1622 (1985); A.D. Stone, ibid. 54, 2692 (1985); B.L. Altshuler and D.E. Khmel'nitskii, Pis'ma Zh. Eksp. Teor. Fiz. 42, 291 (1985) [JETP Lett. 42, 359 (1985)].

${ }^{19}$ R.L. Willet et al., Phys. Rev. Lett. 71, 3846 (1993); W. Kang et al., ibid. 71, 3850 (1993); V.J. Goldman et al., ibid. 72, 2065 (1994).

${ }^{20}$ J.W.C. Beenakker and B. Rejaei, Phys. Rev. Lett. 71, 3689 (1993); J. Chalker and A. Macedo, ibid. 71, 3693 (1993).

21 J.A. Simmons et al., Phys. Rev. B 44, 12933 (1991).

${ }^{22}$ K.B. Efetov, Adv. Phys. 32, 51 (1983).

${ }^{23}$ A.I. Larkin and D.E. Khmel'nitskii, Zh. Eksp. Teor. Fiz. 91, 1815 (1986) [Sov. Phys. JETP 64, 1075 (1986)]; P.A. Lee et al., Phys. Rev. B 35, 1039 (1987); V.I. Fal'ko and D.E. Khmel'nitskii, Zh. Eksp. Teor. Fiz. 95, 328 (1989) [Sov. Phys. JETP 68, 186 (1989)].

${ }^{24}$ It is possible that the formulation of boundary conditions to the diffusion of composite fermions should be extended to some more general form, $\nabla P\left(\mathbf{r}_{s}\right)-P\left(\mathbf{r}_{s}\right) / \lambda=0$. This accounts for a possible decay of a composite quasiparticle in those regions of a sample where the electron density drops and the structure of elementary excitations drastically changes, which would reduce the amplitude of fluctuations.

${ }^{25}$ C.L. Kane et al., Phys. Rev. B 38, 2995 (1988); D.P DiVincenzo and C.L. Kane, ibid. 38, 3006 (1988).

${ }^{26}$ J.K. Jain et al., Phys. Rev. Lett. 71, 3003 (1993). 